This Section of Epidemiology and Psychiatric Sciences regularly appears in each issue of the Journal to cover methodological aspects related to the design, conduct, reporting and interpretation of clinical and epidemiological studies. The aim of these Editorials is to help developing a more critical attitude towards research findings published in international literature, promoting original research projects with higher methodological standards, and implementing the most relevant results of research in every-day clinical practice.

Corrado Barbui, Section Editor and Michele Tansella, Editor EPS

\title{
What is a Cochrane review?
}

\author{
A. Cipriani $^{1 *}$, T. A. Furukawa ${ }^{2}$ and C. Barbui ${ }^{1}$ \\ ${ }^{1}$ Department of Public Health and Community Medicine, Section of Psychiatry and Clinical Psychology, University of Verona, Verona, Italy \\ ${ }^{2}$ Kyoto University Graduate School of Medicine/School of Public Health, Kyoto, Japan
}

Systematic reviews carried out by Cochrane Collaboration (an international network of researchers belonging to this independent, not-for-profit organization) are recognized worldwide as the highest standard in evidence-based healthcare. The main reason is that Cochrane reviews follow a common and specific methodology to limit bias and random error. In this issue, we highlight the most important methodological features of Cochrane reviews, also reporting details on the editorial process to publish the review in the Cochrane Library.

Received 21 March 2011; Revised 24 March 2011; Accepted 25 March 2011

Key words: Bias, meta-analysis, randomized controlled trial, systematic review.

Cochrane reviews are systematic reviews carried out by an international network of researchers (over 28 000 contributors from more than 100 countries) belonging to an independent, not-for-profit organization called 'The Cochrane Collaboration'. The main aim of the Cochrane Collaboration is to help healthcare providers, policy makers, patients, their advocates and carers make well-informed decisions about human healthcare (http://www.cochrane.org/).

One of the main characteristics that make Cochrane reviews internationally recognized as the highest standard in evidence-based healthcare is that they follow a common and specific methodology to limit bias and random error (Shea et al. 2002). A clearly formulated clinical question is addressed by searching for, collating and then assessing with stringent guidelines, all the existing primary studies on a topic. These

* Address for correspondence: Dr Andrea Cipriani, Department of Public Health and Community Medicine, Section of Psychiatry and Clinical Psychology, University of Verona, Ospedale Policlinico, Piazzale L.A. Scuro 10, 37134 Verona, Italy.

(Email andrea.cipriani@univr.it) strategies include the use of explicit, reproducible criteria in the selection of studies, based on a comprehensive search of all potentially relevant studies, including those that are never published. The inclusion of unpublished data in a systematic review is of utmost importance (Trespidi et al. 2011), because it is known that a high proportion of randomized studies are unpublished and that publication status is directly associated with study outcome (Turner et al. 2008). This effort to minimize publication bias is greatly facilitated by the concerted efforts of the Collaboration to keep up-to-date registries of all the published, semi-published (as in the grey literature such as conference abstracts) and unpublished studies. In Cochrane reviews, all methodological issues are preplanned and detailed in the Methods section of a review protocol, which is peer-reviewed and published before starting the search and the assembly of original studies (predominantly randomized controlled trials, but also sometimes non-randomized observational studies) (Cipriani \& Geddes, 2009). This emphasis on review protocols is explained by the need of assuring that what is done by the review 
team corresponds to what was planned, avoiding data dredging and selective outcome reporting that would inevitably produce misleading findings. This makes the whole process very similar to that followed for studies that employ other research designs. To draft the full-text review, primary research designs and study characteristics are appraised, data are synthesized using a meta-analytical approach (if appropriate and feasible) and results are interpreted and discussed (Purgato et al. 2010).

As an initial step, all Cochrane reviews must be registered as titles within a Cochrane review group. This aspect is essential to be sure that a topic is appropriate for a Cochrane review and to prevent duplication with other ongoing reviews that may address the same or a similar question. In the Cochrane Collaboration there are 52 review groups. Each collaborative review group is made up of researchers sharing a similar interest in a particular healthcare problem or type of problem and its main purpose is to prepare and maintain systematic reviews of the effects of healthcare interventions within the scope of the group. Each collaborative review group has an editorial base and is coordinated by an editorial team. The editorial team is made up of the Coordinating Editor, the Managing Editor and the Trials Search Coordinator and usually each collaborative review group has a register of trials which is located where the editorial base is. The review group's editorial base provides an input to the review process from the very beginning (Fig. 1). Suggested review titles are thoroughly discussed with the review group's editorial team; authors are then encouraged to attend a protocol workshop, as a pre-requisite to the preparation and subsequent publication of a protocol, where the plan of how the review will be carried out is reported and detailed. The protocol is intensively peer-reviewed by both internal and external referees before being accepted and published as a protocol in the Cochrane Library. This is followed by work on the main review, with help available for problems with statistical or methodological issues and with trials searching. The drafted review is peer-reviewed again before being published as a completed review in the Library. Finally, the authors, with the help of the editorial team, are responsible for updating their review regularly (usually about once in every two years). At all stages of the process, the work is carefully checked by members of the editorial team and by external referees to ensure its quality.

The general layout of a Cochrane review usually includes a plain-language summary (a short statement summarizing the review's main findings, specifically aimed at lay people), a structured abstract (a summary of the review, subdivided into sections similar to the main review) and the full text. The text has the

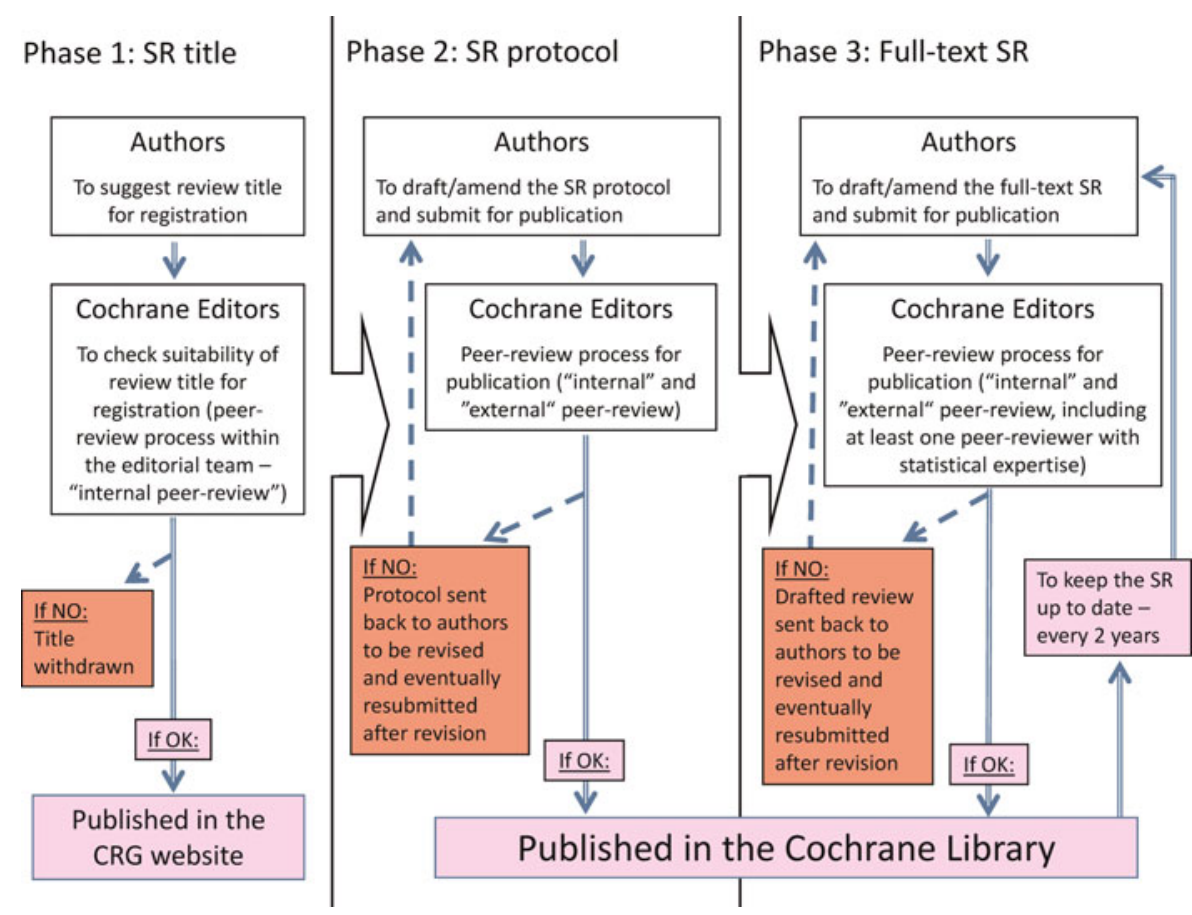

Fig. 1. Different phases of the editorial process to draft and publish a Cochrane review (from title registration to publication of the full-text review). 
following sections: background (an introduction to the question considered, with epidemiological and clinical data, and also explaining the possible mechanism of action of a proposed treatment), objectives (with the aims of the review), the studies' selection criteria (types of studies, types of participants, types of interventions and types of outcome measures), the search strategy (with details on searches of electronic databases, unpublished information, hand-searching of journals or conference proceedings), methods of the review (eligibility for inclusion, how study quality was assessed, how data were extracted from the studies and analyzed, whether any subgroups or sensitivity analyses were carried out), description of characteristics and methodological quality of included studies, results (usually accompanied by forest plots to show meta-analysis results), discussion with interpretation and assessment of results and authors' conclusions (divided into 'Implications for practice' and 'Implications for research'). Cochrane reviews are published in The Cochrane Library, an online collection of databases that brings together in one place rigorous and up-to-date research on the effectiveness of healthcare treatments and interventions, as well as methodology and diagnostic tests (Allen \& Richmond, 2011). Currently there are over 4000 Cochrane Reviews and 2000 protocols available in The Cochrane Library (http://www.thecochranelibrary.com/view/0/index.html). The Library is made freely accessible in many low- to middle-income countries in collaboration with its publisher, Wiley-Blackwell.

\section{References}

Allen C, Richmond K (2011). The Cochrane Collaboration: International activity within Cochrane Review Groups in the first decade of the twenty-first century. Journal of Evidence Based Medicine. doi: 10.1111/j.1756-5391.2011.01109.x. [Epub ahead of print].

Cipriani A, Geddes JR (2009). What is a randomised controlled trial? Epidemiologia e Psichiatria Sociale 18, 191-194.

Purgato M, Barbui C, Cipriani A (2010). Assessing risk of bias in randomized controlled trials. Epidemiologia e Psichiatria Sociale 19, 296-297.

Shea B, Moher D, Graham I, Pham B, Tugwell P (2002). A comparison of the quality of Cochrane reviews and systematic reviews published in paper-based journals. Evaluation and the Health Professions 25, 116-129.

Trespidi C, Barbui C, Cipriani A (2011) Why it is important to include unpublished data in systematic reviews. Epidemiology and Psychiatric Sciences, available at http:// journals.cambridge.org/download.php?file=\%2FEPS\% 2FS2045796011000217a.pdf\&code=2beb2ab475471118ff41ae 708a1ef5e1.

Turner EH, Matthews AM, Linardatos E, Tell RA, Rosenthal $\mathbf{R}$ (2008). Selective publication of antidepressants trials and its influence on apparent efficacy. New England Journal of Medicine 358, 252-260. 